\title{
Stability of commercial phytase sources under different environmental conditions ${ }^{1}$
}

\author{
J. A. De Jong,* J. M. DeRouchey,* M. D. Tokach,* \\ S. S. Dritz, $\uparrow$ R. D. Goodband, $* 2$ J. C. Woodworth, * C. K. Jones, $\$$ and C. R. Stark \\ *Department of Animal Sciences and Industry, College of Agriculture, Kansas State University, Manhattan 66506; \\ $\dagger$ Department of Diagnostic Medicine/Pathobiology, College of Veterinary Medicine, Kansas State University, Manhattan \\ 66506; and $\ddagger$ Department of Grain Sciences and Industry, College of Agriculture, Kansas State University, Manhattan 66506
}

\begin{abstract}
A 300-d study was conducted to evaluate storage stability of 4 commercially available phytase products under varied environmental conditions. Products used were: 1) Quantum Blue G (AB Vista, Plantation, FL), 2) Ronozyme Hi Phos GT (DSM Nutritional Products, Parsippany, NJ), 3) Axtra Phy TPT (Dupont, Wilmington, DE), and 4) Microtech 5000 Plus (Guangdong Vtr Bio-Tech Co., Ltd., Guangdong, China). Products were stored as pure forms, in a vitamin premix, or a vitamin trace mineral (VTM) premix. Pure products were stored at $-20^{\circ} \mathrm{C}, 4^{\circ} \mathrm{C}, 22^{\circ} \mathrm{C}$, or $35^{\circ} \mathrm{C}$ (75\% humidity). Vitamin and VTM premixes were stored at $22^{\circ} \mathrm{C}$ or $35^{\circ} \mathrm{C}$ ( $75 \%$ humidity). Samples were stored in open-topped paper bags and sampled on d 30, $60,90,120,210$, and 300. Stability was determined as the amount of residual phytase activity (\% of initial) at each sampling point. For the pure forms, all interactive and main effects of phytase product, time, and storage temperature were significant $(P<0.05)$. From d 30 to 300 , products had similar reductions in phytase activity at the 3 highest temperatures; however, Quantum Blue G, Ronozyme HiPhos GT, and Axtra Phy TPT had reduced $(P<0.05)$ phytase activity as compared to Microtech 5000 at $-20^{\circ} \mathrm{C}$. In general, as storage time
\end{abstract}

increased, residual phytase activity decreased $(P<0.05)$ regardless of product and storage temperature. When product was stored at $4{ }^{\circ} \mathrm{C}$ and $22^{\circ} \mathrm{C}$, phytase activity was greater than that of product stored at $-20^{\circ} \mathrm{C}$ or $35^{\circ} \mathrm{C}$, and Microtech 5000 Plus had greater $(P<0.05)$ stability regardless of time and temperature as compared to the other 3 products. For vitamin and VTM premixes, a time $\times$ temperature $\times$ product interaction $(P<0.05)$ was observed. When stored at $22^{\circ} \mathrm{C}$, Axtra Phy TPT and Microtech 5000 Plus had reduced residual phytase activity $(P<0.05)$ when compared to the other 2 products; however, when stored at $35^{\circ} \mathrm{C}$ Axtra Phy had even further reduced $(P<0.05)$ activity than the other 3 products regardless of which form the products were stored in. From d 30 to 300 Axtra Phy TPT and Microtech 5000 Plus had the lowest $(P<0.05)$ residual phytase activity when compared to the others regardless of storage form or temperature. Phytase products stored in VTM premix had decreased $(P<0.05)$ residual phytase activity when compared to pure products and vitamin premixes. In conclusion, phytase stored for longer than 90 to $120 \mathrm{~d}$, at both high $\left(35^{\circ} \mathrm{C}\right)$ and low $\left(-20^{\circ} \mathrm{C}\right)$ temperatures when in pure form or as a VTM premix had reduced residual phytase activity.

Key words: phytase, storage, stability, vitamin premix, vitamin trace mineral premix

(C) 2016 American Society of Animal Science. All rights reserved. J. Anim. Sci. 2016.94:4259-4266 doi:10.2527/jas2016-0742

\section{INTRODUCTION}

Currently, phytase sales represent over one-third of the entire feed enzyme market (Yang et al., 2007).

\footnotetext{
${ }^{1}$ Contribution No. 16-208-J from the Kansas Agric. Exp. Stn., Manhattan, KS 66506-0210.

${ }^{2}$ Corresponding author: Goodband@ksu.edu

Received June 22, 2016.

Accepted August 8, 2016.
}

Global expansion in phytase use has led to a large variety of phytases being available in the marketplace. Phytase has repeatedly been shown to improve the digestibility of $\mathrm{P}$ in both swine and poultry (Simons et al., 1990; Lei et al., 1993; Kornegay, 2001). The improved digestibility leads to reduced $\mathrm{P}$ excretion from animals, which reduces the risk of negative impact of livestock on the environmental (Greiner and Konietzny, 2006; Selle and Ravindran, 2007). 
Phytase is susceptible to damage from heat applied to feed during the feed manufacturing process (Jongbloed and Kemme, 1990; Spring et al., 1996). Many phytase manufacturers currently provide products that are claimed to provide protection during pelleting, and thus most research has focused on the ability of phytase to withstand the increased temperature and moisture during the pelleting process (Slominski et al., 2007). However, little research has focused on the shelf life and stability of phytase when stored, especially for longer than 3 to 6 mo. The potential for degradation of phytase from storage conditions or the interaction of phytase with other ingredients in a vitamin or vitamin trace mineral (VTM) premix was reported by Sulabo et al. (2011). Since the publishing of their findings, several manufacturers have released a new generation of phytase products that have not been tested for shelf-life stability. Thus, the objective of our study was to determine the storage stability of 4 commercially available phytase products under varying environmental conditions over $300 \mathrm{~d}$.

\section{MATERIALS AND METHODS}

\section{General}

This study was conducted at the O.H. Kruse Feed Science and Research Center and at Shellenberger Hall at Kansas State University (Manhattan, KS).

\section{Phytase Sources}

This experiment utilized 4 commercially available phytase products: 1) Quantum Blue G (minimum declared concentration of 5,000 FTU/g; AB Vista, Plantation, FL), 2) Ronozyme Hi Phos GT (minimum declared concentration of 2,699 FYT/g; DSM Nutritional Products, Parsippany, NJ), 3) Axtra Phy TPT (minimum declared concentration of 2,500 FTU/g; Dupont, Wilmington, DE), and 4) Microtech 5000 Plus (minimum declared concentration of 5,000 FTU/g; Guangdong Vtr Bio-Tech Co., Ltd., Guangdong, China). One phytase unit (FTU or FYT) was defined as the amount of enzyme that catalyzes the release of $1 \mu \mathrm{mol}$ of iP per minute from $5.1 \mathrm{mM}$ sodium phytate in pH 5.5 buffer at $37^{\circ} \mathrm{C}$ (AOAC 30024; AOAC, 2009). Each phytase product was obtained through a third party distributor. Quantum Blue G and Microtech 5000 Plus are bacterial (E. coli) phytases that are expressed in trichoderma reesie and pichia pastoris, respectively. They are marketed as heat stable phytases, but are not coated. Ronozyme Hi Phos GT is a coated fungal phytase from Citrobacter braakii expressed in aspergillus oryzae. Axtra Phy TPT is a coated phytase from
Table 1. Composition of the vitamin and vitamin trace mineral (VTM) premixes ${ }^{1}$

\begin{tabular}{lcc}
\hline \hline Item & Vitamin premix & VTM premix \\
\hline Vitamin, unit/kg & & \\
Vitamin A, IU & $4,408,000$ & $2,644,800$ \\
Vitamin D, IU & 551,000 & 330,600 \\
Vitamin E, IU & 17,632 & 10,579 \\
Menadione, mg & 1763 & 1058 \\
Vitamin B12, mg & 15 & 9 \\
Niacin, mg & 19,836 & 11,902 \\
Pantothenic acid, mg & 11,020 & 6612 \\
Riboflavin, mg & 3306 & 1984 \\
Trace Mineral, mg/kg & & \\
Cu $\left(\mathrm{CuSO}_{4}\right)$ & - & 3960 \\
I $\left[\mathrm{Ca}\left(\mathrm{IO}_{3}\right)_{2}\right]$ & - & 71 \\
$\mathrm{Fe}\left(\mathrm{FeSO}_{4}\right)$ & - & 26,280 \\
$\mathrm{Mn}\left(\mathrm{MnO}^{2}\right)$ & - & 7920 \\
$\mathrm{Se}\left(\mathrm{NaSeO}_{2}\right)$ & - & 71 \\
$\mathrm{Zn}\left(\mathrm{ZnSO}_{4}\right)$ & - & 26,280 \\
\hline
\end{tabular}

${ }^{1}$ The amount added for each phytase source was determined such that including $0.25 \%$ vitamin premix and $0.4 \%$ VTM would provide 0.12 digestible $\mathrm{P}$ in the diet as noted by the manufacturers recommendations (136,000 and 85,000 FTU/kg, Quantum Blue G [AB Vista, Plantation, FL]; 218,000 and 140,000 FYT/kg, Ronozyme Hi Phos GT [DSM Nutritional Products, Parsippany, NJ]; 141,000 and 90,000 FTU/kg, Axtra Phy TPT [Dupont, Wilmington, DE]; and 318,000 and 203,000 FTU/kg, Microtech 5000 Plus [Guangdong Vtr Bio-Tech Co., Ltd., Guangdong, China] for the vitamin and VTM premixes, respectively).

${ }^{2}$ Vitamin forms were: A (vitamin A acetate, D (cholecalciferol), E (dl$\alpha$-tocophorol acetate), K (menadione dimethylpyrimidinol bisulfite), B12 (cyanocobalamin), niacin (nicotinic acid), pantothenic acid (d-calcium pantothenate), and riboflavin (crystalline riboflavin).

buttiauxella bacterium that is expressed in trichoderma reesei.

\section{Pure Products}

On $\mathrm{d} 0,1.8 \mathrm{~kg}$ of each of the pure phytase products were placed into 8 open, single-lined paper bags. Two bags (observations) of each product were stored in a freezer $\left(-20^{\circ} \mathrm{C}\right)$, in a refrigerator $\left(4^{\circ} \mathrm{C}\right)$, at room temperature $\left(22^{\circ} \mathrm{C}\right)$, and in a controlled environment chamber set at $35^{\circ} \mathrm{C}$ and $75 \%$ humidity. At sampling, approximately $50 \mathrm{~g}$ was removed from each bag on $\mathrm{d} 0,30,60,90,120$, 210 , and 300 . Samples were immediately sent to a commercial facility (New Jersey Feed Labs, Trenton, NJ) for phytase analysis (method 30024; AOAC, 2009).

\section{Premixes}

Each phytase product was added and mixed with either a vitamin premix or a VTM premix (Table 1). The amount added for each phytase product was determined such that including $0.25 \%$ vitamin premix and $0.4 \%$ VTM would provide 0.12 digestible $\mathrm{P}$ in the diet as noted by the manufacturer's recommendations (350 FTU/kg, 
Table 2. Calculated and analyzed phytase value on $\mathrm{d} 0^{1}$

\begin{tabular}{|c|c|c|c|}
\hline Item & 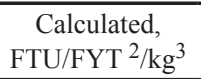 & $\begin{array}{c}\text { Analyzed, } \\
\text { FTU/kg }\end{array}$ & Ratio $^{4}$ \\
\hline \multicolumn{4}{|l|}{ Pure product } \\
\hline Quantum Blue $\mathrm{G}^{5}$ & 5000,000 & 5195,000 & 1.04 \\
\hline Ronozyme Hi Phos GT ${ }^{6}$ & 2700,000 & 4420,000 & 1.64 \\
\hline Axtra Phy TPT ${ }^{7}$ & 2500,000 & 2510,000 & 1.00 \\
\hline Microtech 5000 plus $^{8}$ & 5000,000 & 8055,000 & 1.61 \\
\hline \multicolumn{4}{|l|}{ Vitamin premix } \\
\hline Quantum Blue G & 136,000 & 113,000 & 0.83 \\
\hline Ronozyme Hi Phos GT & 218,000 & 422,000 & 1.94 \\
\hline Axtra Phy TPT & 141,000 & 145,500 & 1.03 \\
\hline Microtech 5000 plus & 318,000 & 584,000 & 1.84 \\
\hline \multicolumn{4}{|c|}{ Vitamin and trace mineral premix } \\
\hline Quantum Blue G & 85,000 & 62,500 & 0.74 \\
\hline Ronozyme Hi Phos GT & 140,000 & 155,500 & 1.11 \\
\hline Axtra Phy TPT & 90,000 & 33,500 & 0.37 \\
\hline Microtech 5000 plus & 203,000 & 327,500 & 1.61 \\
\hline
\end{tabular}

${ }^{1}$ Values represent means of 2 replicate samples each analyzed in duplicate (method 30024; AOAC 2009; New Jersey Feed Labs, Trenton, NJ).

${ }^{2} \mathrm{FTU} / \mathrm{FYT}=$ phytase units.

${ }^{3}$ Calculated values were determined from manufacturers guaranteed minimum.

${ }^{4}$ Analyzed to calculated ratio.

${ }^{5} \mathrm{AB}$ Vista, Plantation, FL.

${ }^{6}$ DSM Nutritional Products, Parsippany, NJ.

${ }^{7}$ Dupont, Wilmington, DE.

${ }^{8}$ Guangdong Vtr Bio-Tech Co., Ltd., Guangdong, China.

Quantum Blue G; 550 FYT/kg, Ronozyme Hi Phos GT; 375 FTU/kg, Axtra Phy TPT; and 850 FTU/kg, Microtech 5000 Plus). Vitamin or VTM premixes were mixed at the aforementioned levels with each phytase source using a $10 \mathrm{~kg}$ ribbon mixer. Each 2-kg batch was then placed in open, single-lined paper bags. Two bags of vitamin and VTM premix with each phytase product were stored at room temperature $\left(22^{\circ} \mathrm{C}\right)$ or in the environmentally controlled chamber set at $35^{\circ} \mathrm{C}$ and $75 \%$ humidity. Sampling occurred on d $0,30,60,90,120,210$, and 300 at which point samples were immediately sent to the same laboratory used for phytase analysis of the pure samples. Each sample weighed approximately $200 \mathrm{~g}$.

\section{Statistical Analysis}

Data were analyzed using a mixed model (MIXED procedure; SAS Inst. Inc., Cary, NC) to determine the interactive and main effects of storage temperature and time on the activity of 4 commercially available phytase products. Because the vitamin and VTM premixes were stored only at room temperature and in the environmentally controlled heat chamber, 2 analyses were performed. The first analysis was with the pure forms only. The second analysis was for the pure forms, vitamin premixes, and VTM premixes stored at $22^{\circ} \mathrm{C}$ and $35^{\circ} \mathrm{C}$. When treatment was a significant source of variation, differences were determined by using the preplanned pairwise comparisons. Results were considered significant at $P \leq 0.05$ and considered marginally significant between $P>0.05$ and a tendency at $P \leq 0.10$.

\section{RESULTS AND DISCUSSION}

The initial ( $\mathrm{d}$ 0) calculated and analyzed phytase activities and the ratio of analyzed to calculated of the pure products, vitamin premix, or VTM premixes are reported in Table 2. Calculated values were determined from the minimum declared phytase concentrations as provided by each product's manufacturer. For the pure products, phytase activity ranged from 1.00 for Axtra Phy TPT to 1.64 times greater than the calculated values for Ronozyme Hi Phos GT. For the vitamin premixes, phytase activity ranged from 0.83 for Quantum Blue $\mathrm{G}$ to 1.84 times greater than the calculated values for Microtech 5000 Plus. In regards to the VTM premixes, phytase values ranged from 0.37 for Axtra Phy TPT to 1.61 times greater than the calculated values for Microtech 5000 Plus. There may be several reasons for the differences observed between analyzed and calculated concentrations of phytase. It has been shown that the actual concentration of the product may be greater than the minimum guarantee (Sulabo et al., 2011). Second, it has been shown that there is large analytical variation associated with the laboratory assays utilized to measure phytase activity (Jones et al., 2010). In addition, the differences in the coating methods among the products may have inherently led to differences in assay results. Ronozyme HiPhos GT, Axtra Phy TPT, and Microtech 5000 Plus all utilize a fat-based coating which may interfere with the AOAC (2009) lab assay. However, more research is necessary to determine if product coating can affect lab assay. Finally, the minimum declared concentrations are determined using internal assays from each company which in general are variations on the official AOAC method used in the current experiment (method 30024; AOAC, 2009). These internal assays were used to provide concentrations for the calculated values. Thus, differences may have been present when comparing the calculated to analyzed values for $\mathrm{d} 0$ due to variations in the analysis method.

De Jong et al. (2015) observed that a $75^{\circ} \mathrm{C}$ conditioning temperature resulted in residual phytase activity of $21 \%$ to $78 \%$, while a $95^{\circ} \mathrm{C}$ conditioning temperature resulted in residual phytase activity ranging from $3 \%$ to $33 \%$. This indicates that at normal conditioning temperatures, phytase products may be affected by added heat and moisture. They also noted that Microtech 5000 Plus had less activity regardless of pelleting temperature when compared to Quantum Blue G, Ronozyme 
Table 3. Probabilities of interactive and main effects of storage time, temperature, and phytase product on stability (as defined by percentage of initial phytase activity) of phytase products in pure forms

\begin{tabular}{ll}
\hline \hline Item & Probability, $P<$ \\
\hline Interactive effect & \\
Time $\times$ temperature $\times$ product & 0.001 \\
Time $\times$ temperature & 0.001 \\
Time $\times$ product & 0.001 \\
Temperature $\times$ product & 0.001 \\
Main effect & \\
Time & 0.001 \\
Temperature & 0.001 \\
Product & 0.001 \\
\hline
\end{tabular}

HiPhos GT, or Axtra Phy TPT. In the current experiment, Microtech 5000 Plus and Ronozyme HiPhos GT had greater $(P<0.05)$ stability $(96 \%$ and $94 \%)$ regardless of time and temperature as compared to Quantum Blue G and Axtra Phy TPT (91\% and 90\%, respectively; Table 3; Fig. 1). It appears that stability during pellet conditioning does not correspond to stability during storage, especially in regards to Microtech 5000 Plus.

Phytase is exposed to varied temperatures and humidity levels during storage. Depending on location and season, phytase may be exposed to temperatures up to $35^{\circ} \mathrm{C}$ and relative humidity of $75 \%$ to $90 \%$ during summer. Sulabo et al. (2011) previously reported that when stored at $23^{\circ} \mathrm{C}$ or less, pure phytase products retained the most phytase activity when stored for 120 d. This also agrees with results of research evaluating other enzymes by El-Sherbiny and El-Chaghaby (2011), who found that a liquid enzyme mix (xylanase, amylase, and cellulose) had greater stability when stored at $4{ }^{\circ} \mathrm{C}$ as compared to $30^{\circ} \mathrm{C}$. In the current experiment, products had similar reductions in phytase activity from $\mathrm{d}$ 30 to 300 at the 3 highest temperatures; however, for Quantum Blue G, Ronozyme HiPhos GT, and Axtra Phy TPT (58\%, 53\%, 66\%) phytase activity decreased $(P<0.05)$ as compared to Microtech $5000(83 \%)$ on d 300 when stored at $-20^{\circ} \mathrm{C}$. Also, when products were stored at $4^{\circ} \mathrm{C}$ or $22^{\circ} \mathrm{C}$, phytase activity was more stable (99\% and $97 \%$ ) compared to product stored at $-20^{\circ} \mathrm{C}$ or $35^{\circ} \mathrm{C}(87 \%$ and $89 \%)$. Pure product stored at the lowest temperature $\left(-20^{\circ} \mathrm{C}\right)$ had worse stability than pure product stored at the highest temperature. This finding may have been an artifact of the freezing and thawing that occurred during sampling throughout the duration of the trial. Cao et al. (2003) noted that proteins were more susceptible to denaturation and destruction when exposed to fast freezing and slow thawing in an aqueous solution. The authors noted that fast-freezing proteins are more exposed to ice crystals which can lead to protein degradation. This could have occurred dur-
Table 4. Probabilities of interactive and main effects of storage time, temperature, and phytase product on stability (as defined by percentage of initial phytase activity) of phytase products

\begin{tabular}{ll}
\hline \hline Item & Probability, $P<$ \\
\hline Interactive effect & \\
Time $\times$ temperature $\times$ product $\times$ form & 0.123 \\
Time $\times$ temperature $\times$ product & 0.018 \\
Time $\times$ temperature $\times$ form & 0.932 \\
Temperature $\times$ product $\times$ form & 0.138 \\
Time $\times$ temperature & 0.750 \\
Time $\times$ product & 0.001 \\
Time $\times$ form & 0.001 \\
Temperature $\times$ product & 0.001 \\
Temperature $\times$ form & 0.049 \\
Form $\times$ product & 0.001 \\
Main effect & \\
Time & 0.001 \\
Temperature & 0.001 \\
Form & 0.001 \\
Product & 0.001 \\
\hline
\end{tabular}

ing sampling in the current experiment as samples were removed from the freezer to weigh and sample product which inevitably led to slight amounts of thawing and subsequent re-freezing. Sulabo et al. (2011) also stored products at similar low temperatures but didn't observe negative results of freeze storing as observed in the current experiment. The difference may be due in part to the storage protocols used in their study that allowed product to remain in the freezer until sampling occurred unlike the current experiment where samples were removed every time sampling occurred. In addition, Greiff and Kelly (1966) and Whittam and Rosano (1973) showed that continued freezing and thawing reduced enzyme activity of lactic dehydrogenase and $\alpha$ amylase, respectively. Thus, this may have occurred with the phytase products used in the present study.

In addition, Sulabo et al. (2011) also suggested that the reduced phytase activity may have been attributed to the $75 \%$ humidity employed at the highest temperature. Yang et al. (2007) showed that as humidity was increased from $53 \%$ to $89 \%$, phytase activity decreased. Iyer and Ananthanarayan (2008) also noted that moisture can lower the temperature required to unfold an enzyme, suggesting that added moisture at the highest temperature herein may have added to the denaturation of the phytase enzyme. In general, it appears that storing phytase products at temperatures below $35^{\circ} \mathrm{C}$ and above $-20^{\circ} \mathrm{C}$ and at low humidity levels will result in the greatest phytase retention.

Under commercial conditions phytase is stored at variable temperatures and for varying lengths of time. Though most feed mills ensure turnover of their micro- 

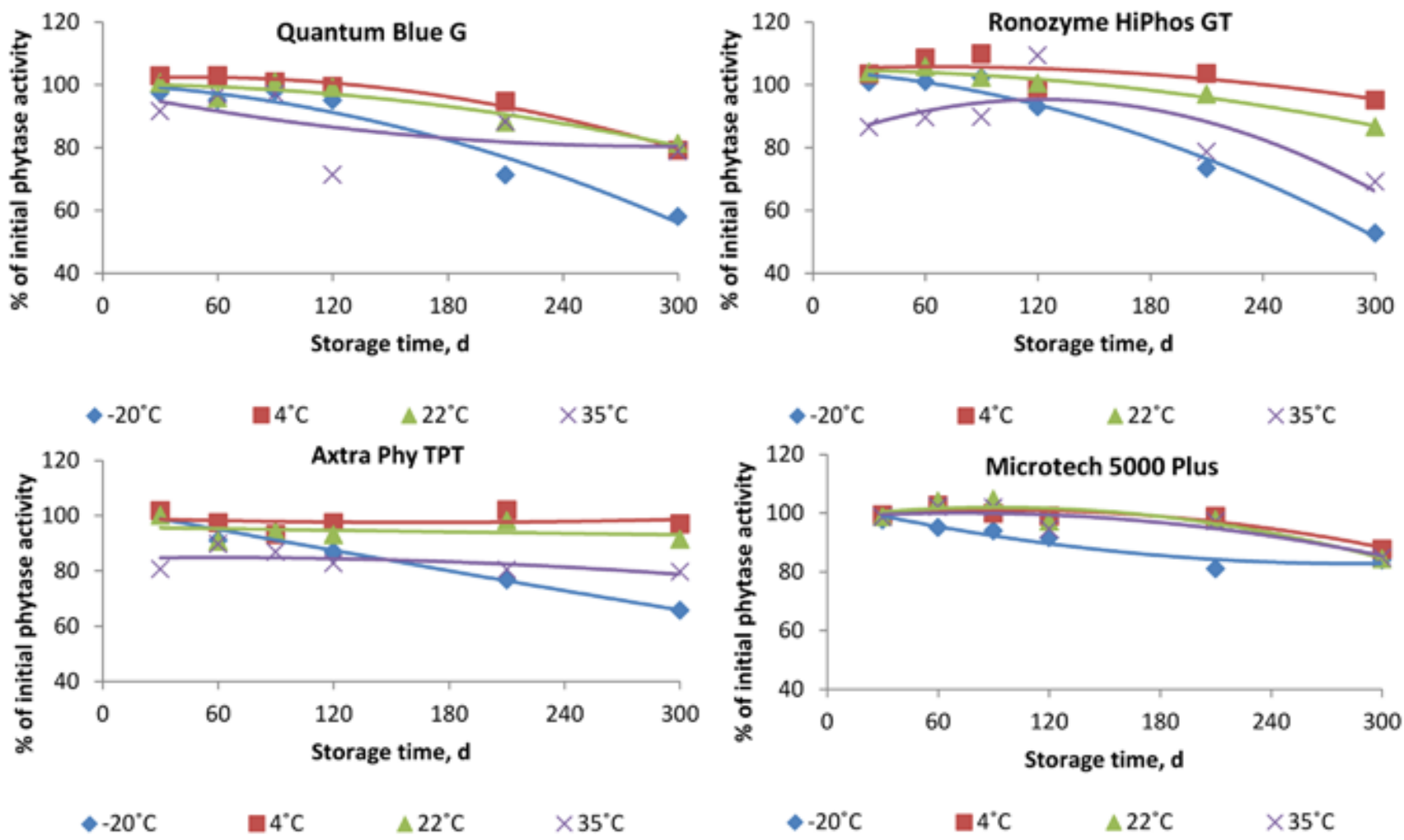

Figure 1. Residual phytase activity (\% of initial) for Quantum Blue G (AB Vista, Plantation, FL), Ronozyme HiPhos GT (DSM Nutritional Products, Parsippany, NJ), Axtra Phy TPT (Dupont, Wilmington, DE), and Microtech 5000 Plus (Guangdong Vtr Bio-Tech Co., Ltd., Guangdong, China) as affected by storage temperature (freezer $\left[-20^{\circ} \mathrm{C}\right]$, refrigerator $\left[4^{\circ} \mathrm{C}\right]$, room temperature $\left[22^{\circ} \mathrm{C}\right]$, and controlled environment chamber $\left[35^{\circ} \mathrm{C}\right.$ and $75 \%$ humidity]) and time ( 30 to $300 \mathrm{~d}$ ). Each data point is the mean of 2 observations

ingredients within 3 to 6 mo after receiving them, it is possible that product may remain in storage for long periods of time in low volume mills. It is also possible that sudden dietary changes may require less of an ingredient, such as phytase, resulting in increased storage time prior to use. In the present study, as storage time increased, residual phytase activity decreased $(P<0.05)$ from $\mathrm{d}$ 30 to $300(98 \%$ to $80 \%)$ regardless of product and storage temperature. Sulabo et al. (2011) also reported that

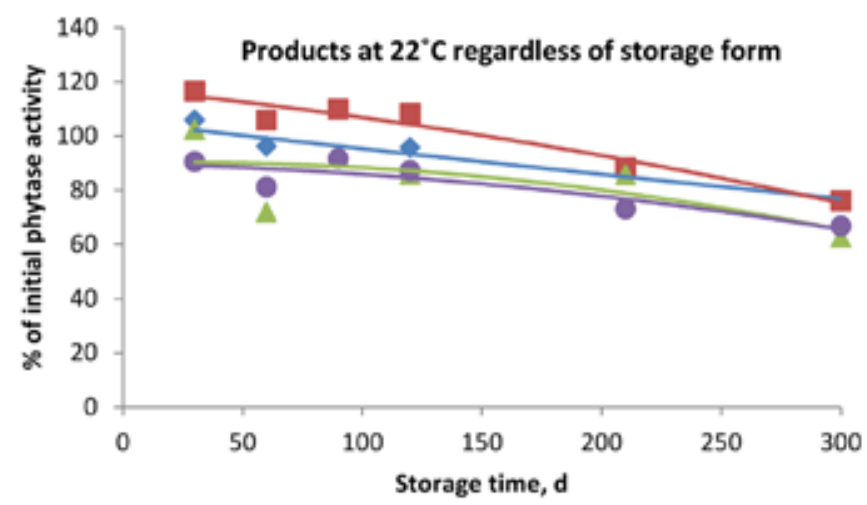

\footnotetext{
$\downarrow 22^{\circ} \mathrm{C}$ Quantum Blue G

$\triangle 22^{\circ} \mathrm{C}$ Axtra Phy TPT
}

phytase stored as pure product had significantly reduced phytase activity when stored for as little as $60 \mathrm{~d}$. Lu et al. (2013) and Naves et al. (2012) both found that when pure product was stored for $180 \mathrm{~d}$, phytase activity was reduced by $25 \%$ and $67 \%$, respectively. They speculated that decreases in phytase activity over time may be attributed to the natural proteolytic degradation of the peptide ring during storage as a result of the increased temperatures or exposure to oxygen radicals. More recently,

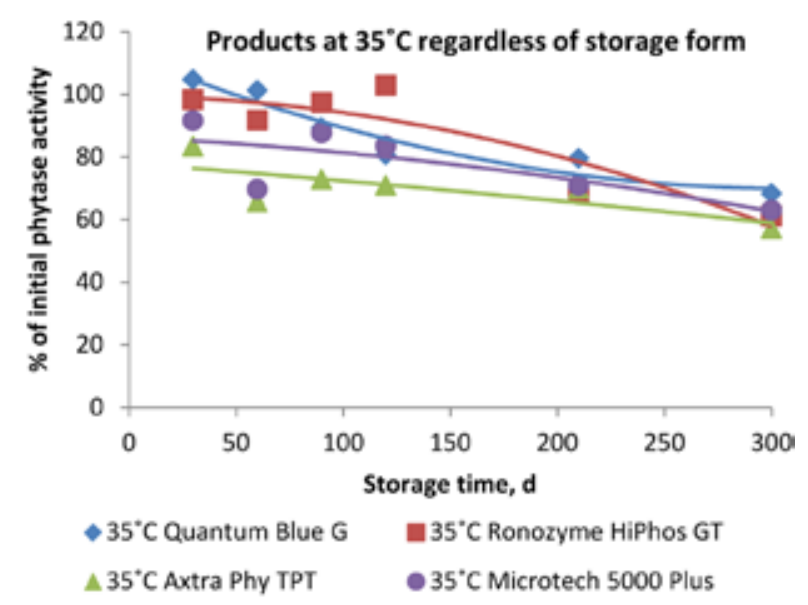

Figure 2. Residual phytase activity (\% of initial) for Quantum Blue G (AB Vista, Plantation, FL), Ronozyme Hi Phos GT (DSM Nutritional Products, Parsippany, NJ), Axtra Phy TPT (Dupont, Wilmington, DE), and Microtech 5000 Plus (Guangdong Vtr Bio-Tech Co., Ltd., Guangdong, China) as affected by storage temperature $\left(22^{\circ} \mathrm{C}\right.$ and $35^{\circ} \mathrm{C}$ ) and time ( 30 to $\left.300 \mathrm{~d}\right)$ and regardless of storage form. Each data point is the mean of 6 observations. 


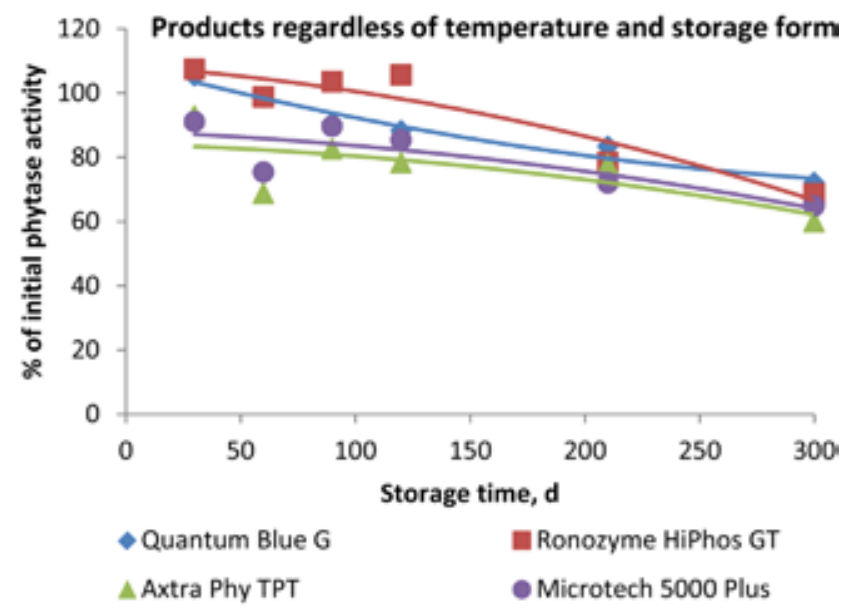

Figure 3. Residual phytase activity (\% of initial) for Quantum Blue G (AB Vista, Plantation, FL), Ronozyme Hi Phos GT (DSM Nutritional Products, Parsippany, NJ), Axtra Phy TPT (Dupont, Wilmington, DE), and Microtech 5000 Plus (Guangdong Vtr Bio-Tech Co., Ltd., Guangdong, China) as affected by time ( 30 to $300 \mathrm{~d}$ ) and regardless of storage form and temperature. Each data point is the mean of 12 observations.

the stability of Ronozyme HiPhos GT was reported (European Food Safety Authority, 2012) to retain $>90 \%$ activity when stored for 18 mo at 5 temperatures $\left(-18^{\circ} \mathrm{C}\right.$, $10^{\circ} \mathrm{C}, 25^{\circ} \mathrm{C}, 35^{\circ} \mathrm{C}$, and $40^{\circ} \mathrm{C}$ ). This disagrees with the current experiment where Ronozyme HiPhos GT had $>90 \%$ retention only when stored at $4^{\circ} \mathrm{C}$ and $22^{\circ} \mathrm{C}$. Phytase retention was similar from d 0 to 120 for pure product stored at $-20^{\circ} \mathrm{C}$ or $35^{\circ} \mathrm{C}$ but was reduced to $52 \%$ and $59 \%$ at $\mathrm{d} 300$. The differences in the current data and data from European Food Safety Authority (2012) are currently unknown but may have been caused by the thawing and re-freezing at the lowest temperatures and the high humidity utilized at the highest temperature. Both experiments found reductions over time in phytase activity regardless of temperature. In general, it appears that time, in combination with moisture and temperature, may lead to protein degradation minimizing pure product's efficacy during extended storage.

When stored at $22^{\circ} \mathrm{C}$, Axtra Phy TPT and Microtech 5000 Plus's residual phytase decreased $(P<0.05)$ when compared to the other 2 products (Table 4 ) regardless of storage form. However, when stored at $35^{\circ} \mathrm{C}$, Axtra Phy TPT phytase activity decreased more $(P<0.05)$ than the other 3 products (Fig. 2). It appears that Axtra Phy TPT was less stable at high temperatures and long storage periods than the other 3 products. In addition, from d 30 to 300, Axtra Phy TPT and Microtech 5000 Plus had the lowest $(P<0.05)$ residual phytase activity when compared to the other 2 products regardless of storage form and temperature (Fig. 3). This disagrees with the current data for pure products where Microtech 5000 Plus was the most stable. The presence of vitamins or vitamins and trace minerals in the premixes appear to have had a larger degrading effect on Axtra Phy

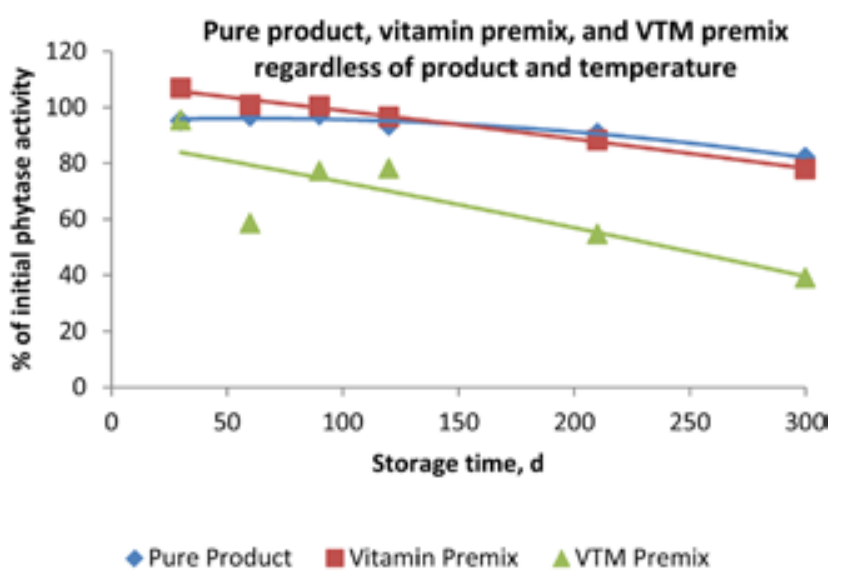

Figure 4. Residual phytase activity (\% of initial) for phytase stored as pure phytase, vitamin premix, and VTM premix as affected by time ( 30 to $300 \mathrm{~d}$ ), regardless of product and temperature. Each data point is the mean of 16 observations.

TPT and Microtech 5000 Plus. This is especially true for Microtech 5000 Plus as it was the most stable product when stored in pure form and one of the least stable when stored as a vitamin or VTM premix.

In general, phytase activity decreased $(P<0.05)$ when product was stored as a VTM premix when compared to the pure product and vitamin premixes (Fig. 4). This could have resulted from phytase and micro-ingredients present in the VTM premix negatively interacting with each other. Greater destruction of phytase when stored as part of a VTM premix as compared to the pure product or a vitamin premix has been previously observed by both Sulabo et al. (2011) and Naves et al. (2012). Both reported lower phytase activities throughout their storage studies when phytase was stored as part of a VTM premix as compared to either pure phytase or product stored as a vitamin premix. Lu et al. (2013) speculated that the reduced phytase activity in VTM premixes may have been caused by the $\mathrm{Cu}$ product used in the premix. Thus, they stored phytase with either tribasic copper chloride (TBCC) or $\mathrm{CuSO}_{4}$ for 0 to $40 \mathrm{~d}$ and at 3 temperatures $\left(23^{\circ} \mathrm{C}, 32^{\circ} \mathrm{C}\right.$, or $\left.38^{\circ} \mathrm{C}\right)$. Phytase stored with TBCC had better retention as compared to phytase stored with $\mathrm{CuSO} 4$, which the authors noted may have been a result of the decreased water solubility and disassociation to form cations of TBCC. This is similar to Liu et al. (2005), who also found that TBCC in a complete poultry feed resulted in higher phytase retention after $21 \mathrm{~d}$ when compared to $\mathrm{CuSO} 4$. In the current study, CuSO4 was used in the VTM premixes which may have led to the decreased phytase retention when compared to the pure products or vitamin premixes. In addition, Chantasartrasamee et al. (2005) also reported that when stored with either $\mathrm{Fe}^{2+}, \mathrm{Ca}^{2+}$, $\mathrm{Mn}^{2+}$, or $\mathrm{Mg}^{2+}$ phytase had similar or even improved activity; however, when stored with $\mathrm{Zn}^{2+}$ phytase activ- 


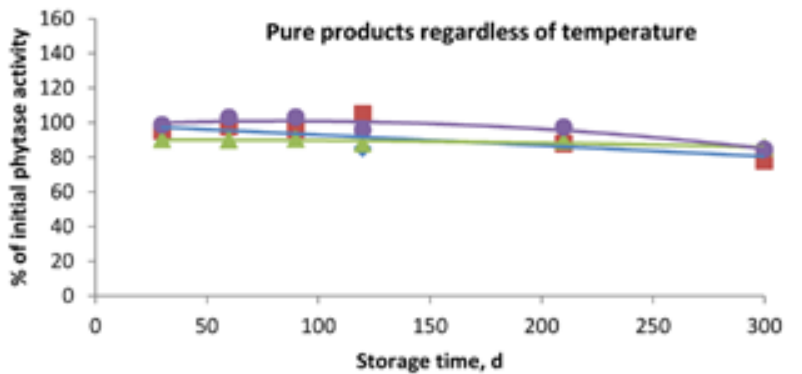

• Quantum Blue G nenozyme HiPhos GT A Axtra Phy TPT e Microtech 5000 Plus

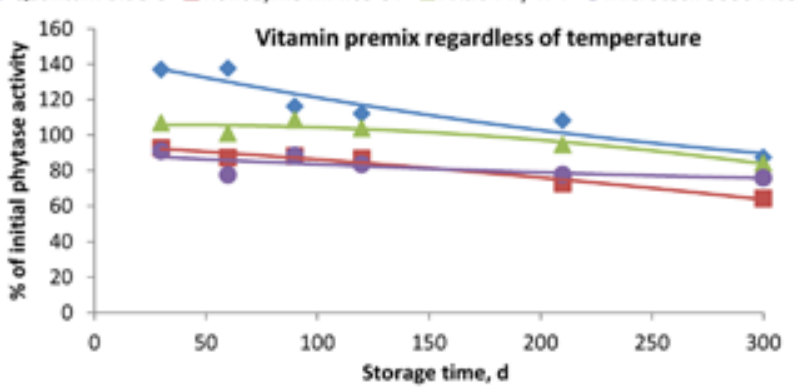

• Quantum Blue G a Ronozyme HiPhos GT $\triangle$ Axtra Phy TPT - Microtech 5000 Plus

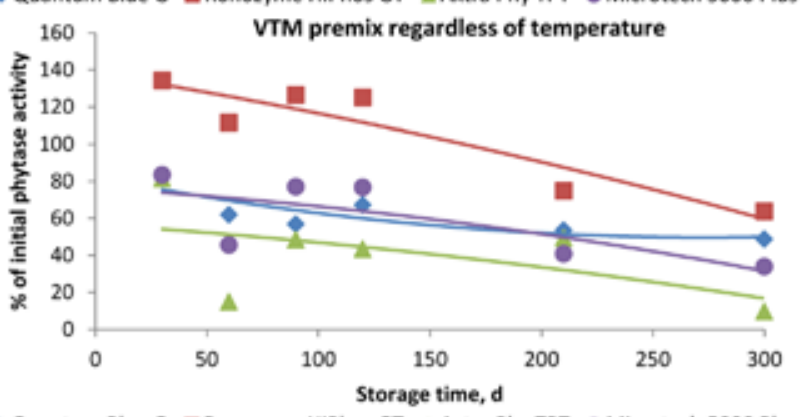

$\bullet$ Quantum Blue G $=$ Ronoryme HiPhos GT $\triangle$ Axtra Phy TPT - Microtech 5000 Plus

Figure 5. Residual phytase activity (\% of initial) for Quantum Blue G (AB Vista, Plantation, FL), Ronozyme Hi Phos GT (DSM Nutritional Products, Parsippany, NJ), Axtra Phy TPT (Dupont, Wilmington, DE), and Microtech 5000 Plus (Guangdong Vtr Bio-Tech Co., Ltd., Guangdong, China) stored in pure form, vitamin premix, or VTM premix regardless of storage temperature. Each data point is the mean of 4 observations.

ity was reduced to levels that were undetectable. The authors speculated $\mathrm{Zn}$ may have been binding with the phytase enzyme, inhibiting its activity. Shurson et al. (2011) also showed that inorganic trace mineral sources were more destructive to vitamins present in the premix as compared to metal-specific AA complexes. Though phytase was not present in their study inorganic mineral sources may also have a more negative effect on phytase storage. It appears that $\mathrm{Cu}, \mathrm{Zn}$, or other inorganic mineral sources may have negative effects on phytase activity when stored in a VTM premix which supports the current findings of the VTM phytase retention.

When stored as a vitamin premix, Quantum Blue and Axtra Phy had greater $(P<0.05)$ residual phytase activity than Ronozyme HiPhos GT and Microtech 5000 Plus (Fig. 5). When stored as a VTM premix Ronozyme HiPhos GT had the greatest $(P<0.05)$ residual phytase activity as compared to the other 3 products. Currently, the reason for differences between phytase products stored in the different forms is unknown. However, Sulabo et al. (2011) reported that coated products were more stable during storage compared to similar, uncoated products. In the current experiment, Quantum Blue G had improved stability as a vitamin premix even though it was the only uncoated product, which disagrees with their results. Although unlikely, it is also possible that phytase coating may be interfering with the assay utilized in the current experiment; however, further research is needed to determine the effect of coating on assay efficacy.

In conclusion, when storing phytase in pure form, it appears residual phytase activity is maximized when stored between $4^{\circ} \mathrm{C}$ and $22^{\circ} \mathrm{C}$. When phytase was stored as part of a VTM premix residual phytase activity was reduced by the greatest magnitude compared to the pure form and vitamin premix. In general phytase stored for longer than 90 to $120 \mathrm{~d}$, at high or low temperatures in pure form, or as a VTM premix, had reduced residual phytase activity. Procedures should be in place to ensure that phytase storage time prior to feed manufacturing is minimized and products are stored at temperatures between $4^{\circ} \mathrm{C}$ and $22^{\circ} \mathrm{C}$ to minimize degradation and ensure phytase levels in complete feed are similar to those used in formulation. Use of phytase in a VTM premix should be discouraged unless excess phytase is added to overcome the large loss in phytase activity.

\section{LITERATURE CITED}

AOAC. 2009. Official methods of analysis. 18th ed. Assoc. Off. Anal. Chem., Gaithersburg, MD.

Cao, E., Y. Chen, Z. Cui, and P. R. Foster. 2003. Effect of freezing and thawing rates on denaturation of proteins in aqueous solutions. Biotechnol. Bioeng. 82:684-690.

Chantasartrasamee, K., D. I. Na Ayuthaya, S. Intarareugsorn, and S. Dharmsthiti. 2005. Phytase activity from Aspergillus oryzae AK9 cultivated on solid state soybean meal medium. Process Biochem. 40:2285-2289.

De Jong, J. A., J. C. Woodworth, J. M. DeRouchey, R. D. Goodband, M. D. Tokach, S. S. Dritz, C. R. Stark, and C. K Jones. 2015. Stability of four commercial phytase products under increasing thermal conditioning temperatures. J. Anim. Sci. 94(Suppl. 2):101. (Abstr.)

El-Sherbiny, M. A., and G. A. El-Chaghaby. 2011. Storage temperature and stabilizers in relation to the activity of commercial liquid feed enzymes: A case study from Egypt. J. Agrobiol. 28:129-137.

European Food Safety Authority. 2012. Scientific opinion on the safety and efficacy of Ronozyme HiPhos GT (6-phytase) as feed additive for poultry and pigs. EFSA J. 10(6):2730.

Greiff, D., and R.T. Kelly. 1966. Cryotolerance of enzymes: I. Freezing of lactic dehydrogenase. Cryobiology 2:335-341.

Greiner, R. U., and U. Konietzny. 2006. Phytase for food application. Food Technol. Biotechnol. 44:125-140.

Iyer, P. V., and L. Ananthanarayan. 2008. Enzyme stability and stabilization-Aqueous and non-aqueous environment. Process Biochem. 43:1019-1032. 
Jongbloed, A. W., and P. A. Kemme. 1990. Effect of pelleting mixed feeds on phytase activity and the apparent absorbability of phosphorus and calcium in pigs. Anim. Feed Sci. Technol. 28:233242.

Jones, C. K., M. D. Tokach, S. S. Dritz, B. W. Ratliff, N. L. Horn, R. D. Goodband, J. M. DeRouchey, R. C. Sulabo, and J. L. Nelssen. 2010. Efficacy of different commercial phytase enzymes and development of an available phosphorus release curve for Escherichia coli-derived phytases in nursery pigs. J. Anim. Sci. 88:3631-3644.

Kornegay, E. T. 2001. Digestion of phosphorus and other nutrients: The role of phytases and factors influencing their activity. In: M. R. Bedford, and G. G. Partridge, editors, Enzymes in farm animal nutrition, CABI Publishing, Wallingford, UK. p. 237-271.

Lei, X. G., P. K. Ku, E. R. Miller, M. T. Yokoyama, and D. E. Ullrey. 1993. Supplementing corn-soybean meal diets with microbial phytase maximizes phytate phosphorus utilization by weanling pigs. J. Anim. Sci. 71:3368-3375.

Liu, Z., M. M. Bryant, and D. A. Roland. 2005. Layer performance and phytase retention as influenced by copper sulfate pentahydrate and tribasic copper chloride. J. Appl. Poult. Res. 14:499-505.

Lu, L., S. Hao, L. Zhang, and X. Luo. 2013. Effect of copper source on phytase stability in the premix of weanling piglets. Anim. Prod. Sci. 53:142-145.

Naves, L. P., A. D. Correa, A. G. Bertechini, and E. M. Gomide. 2012. Activity of fungal phytases stored in two ways in response to the period of storage at room temperature. Cienc. Rural 42:178-183.
Selle, P. H., and V. Ravindran. 2007. Microbial phytase in poultry nutrition. Anim. Feed Sci. Technol. 135:1-41.

Shurson, G. C., T. M. Salzer, D. D. Koehler, and M. H. Whitney. 2011. Effect of metal specific amino acid complexes and inorganic trace minerals on vitamin stability in premixes. Anim. Feed Sci. Technol. 163:200-206.

Simons, P. C. M., H. A. J. Versteegh, A. W. Jongbloed, P. A. Keeme, P. Slump, K. D. Bos, M. G. E. Wolters, R. F. Beudeker, and G. J. Verschoor. 1990. Improvement of phosphorus availability by microbial phytase in broilers and pigs. Br. J. Nutr. 64:525-540.

Slominski, B. A., T. Davie, M. C. Nyachoti, and O. Jones. 2007. Heat stability of endogenous and microbial phytase during feed pelleting. Livest. Sci. 109:244-246.

Spring, P., K. E. Newman, V. Wenk, R. Messikommer, and M. Vukic Vranjes. 1996. Effect of pelleting temperature on the activity of different enzymes. Poult. Sci. 75:357-361.

Sulabo, R. C., C. K. Jones, M. D. Tokach, R. D. Goodband, S. S. Dritz, D. R. Campbell, B. W. Ratliff, J. M. DeRouchey, and J. L. Nelssen. 2011. Factors affecting storage stability of various commercial phytase sources. J. Anim. Sci. 89:4262-4271.

Whittam, J. H., and H. L. Rosano. 1973. Effects of the freeze-thaw process on $\alpha$ amylase. Cryobiology 10:240-243.

Yang, H., X. Lu, J. Wang, J. Li, H. Li, and Y. Qin. 2007. Near-infrared reflectance spectroscopy-based methods for phytase registration in feed industry. J. Agric. Food Chem. 55:7667-7675. 